CLINICAL PRACTICE

\title{
Simplifying trauma airway management in South African rural hospitals
}

\author{
M Berry, D Wood \\ Michael Berry is a UK trainee in anaesthesia and intensive care. He worked for a year in the resuscitation unit at Ngwelezane Hospital, KwaZulu- \\ Natal, South Africa. Darryl Wood is an emergency medicine specialist at the University of KwaZulu-Natal and Ngwelezane Hospital. He is currently \\ undertaking a research sabbatical and completing his PhD.
}

Corresponding author: M Berry (michael.berry1@nhs.net)

South African emergency centres witness high levels of trauma. Successfully managing a compromised trauma airway requires considerable skill and expertise. In the rural healthcare setting, clinics and hospitals are often staffed by junior doctors without formal advanced airway training. Current airway management algorithms tend to ignore lack of resources and skill. We therefore propose a simplified guideline for the rural hospital practitioner. Our algorithm offers a step-by-step approach, with the aim of providing an easy sequence to follow that will ensure successful airway management and patient safety. The paucity of advanced airway equipment in most rural hospitals is taken into consideration.

S Afr Med J 2014;104(9):604-606. DOI:10.7196/SAMJ.8064

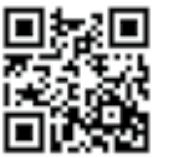

\section{Trauma care in rural emergency centres}

South Africa (SA) is a middle-income country characterised by high levels of trauma. Road traffic accidents and interpersonal violence are common.

This situation is fuelled by rapid urbanisation as well as socioeconomic disparities. ${ }^{[1]}$ The injury burden typically peaks over weekends and on paydays and public holidays. Consequently, many emergency centres are inundated with victims of blunt and penetrating trauma. The annual estimated trauma incidence in KwaZulu-Natal (KZN), the most populous province in SA, is $12.9 / 1000$ population. ${ }^{[2]}$ Comparing these figures with European data, e.g. for London, UK, where the incidence of trauma is around 0.5/1 000, clearly demonstrates the high trauma load in SA. ${ }^{[3]}$

Many trauma patients in KZN present outside urban centres to facilities that are often basic and frequently understaffed. ${ }^{[4]}$ The skill sets of hospital staff vary significantly, with many smaller hospitals commonly relying on inexperienced junior doctors with minimal training and equipment to manage all aspects of major trauma. Ensuring that the airway is patent and protected is the most crucial initial intervention in the treatment of trauma patients. ${ }^{[5]}$

\section{Current practice and its limitations}

The management of the trauma airway can be hugely challenging, and differs significantly from airway emergencies encountered in the operating room in an elective setting. ${ }^{[6]}$ Haemodynamic instability, time pressure, risk of aspiration, the need for cervical spine protection, penetrating neck trauma and facial injuries represent some of the issues that make trauma airway management difficult. ${ }^{[6]}$ Moreover, for similar reasons it is rarely possible to assess the airway comprehensively in an attempt to predict potential difficulties and prepare accordingly. Dealing with such situations requires a level of clinical experience that is often not available in the rural hospital environment. Guidelines presented by the Emergency Medicine Society of South Africa (EMSSA), the Difficult Airway Society (DAS) in the UK and the American Society of Anesthesiologists (ASA) are comprehensive and provide many useful pointers on how to deal with these situations. ${ }^{[7-9]}$ Nevertheless, useful as these guidelines are, in our opinion they fail to address a number of fundamental issues.

Firstly, the guidelines are not specific to the trauma patient requiring prompt intubation. Most discuss a variety of rapidsequence intubation (RSI) techniques and commonly focus on the elective theatre setting. For example, both the ASA and the DAS emphasise waking the patient up following failed intubation, which is seldom appropriate or safe in critically injured trauma patients. ${ }^{[8,9]}$

Secondly, an experienced airway provider is frequently not immediately available to manage a trauma airway. It is well known that under circumstances of acute stress, decision-making and the ability to process new information are severely impaired. ${ }^{[10]}$ Most algorithms have many intermediate steps with confusing amounts of arrows and/or explanatory text spread over a number of pages, which renders them impractical in an emergency. ${ }^{[7-9]}$

Thirdly, both SA and international protocols incorporate airway adjuncts and techniques that are not commonplace in rural emergency centres ${ }^{[7-9]}$ Furthermore, the suggested resort to use of unfamiliar or infrequently used equipment, such as fibreoptic techniques, under pressure seems counterintuitive. ${ }^{[7-9]}$

Finally, supraglottic airway (SGA) devices should be used much earlier and play a key role in rescuing the emergency trauma airway in the hands of inexperienced staff. Initiating bag-mask ventilation or other airway adjuncts prior to using an SGA when oxygenation and ventilation are compromised makes little sense in our opinion, particularly since face-mask ventilation is often done poorly in an emergency situation, increasing the risk of gastric insufflation and pulmonary aspiration. ${ }^{[11]}$

We therefore suggest a single-page practical management guideline taking the above challenges into account. Since the safety of the patient is paramount, the aim is to provide clear guidance to ensure gas exchange in situations where a junior doctor with limited experience is faced with managing the trauma airway.

Our protocol is primarily a modification of the emergency airway management algorithm used at the Shock Trauma Center (STC) of 
the University of Maryland (Baltimore, USA), as well as incorporating elements of the EMSSA protocol. ${ }^{[7,12]}$ It is a simplification of these guidelines and adapted for use in the rural hospital trauma setting, in a situation where loss of the airway is imminent and the option to reverse the anaesthetic, as well as awaken the patient, is not possible. The protocol takes into account that the operator may only have basic skills. Aware that equipment is often rudimentary, we elected not to include adjuncts more advanced than a bougie, SGA devices and tracheostomy tubes. We designed our algorithm seeking procedural simplicity and utilising minimum recommended equipment for an emergency centre, as suggested by EMSSA. ${ }^{[13]}$ The algorithm is illustrated in Fig. 1 and provides a step-by-step approach to managing a compromised trauma airway in the rural healthcare setting.

In opting for visual clarity, we used a single-page layout with a simple three-step approach (intubation - rescue device - surgical airway) outlined on the left-hand side of the page. The decision tree in our guideline is limited to two branches, which greatly reduces the number of decisions that a doctor needs to make in a stressful situation, allowing it to be used at the patient's bedside in an emergency.

A similar three-step approach is integral to the STC protocol and has been validated in a study of 6088 emergency trauma intubations over a 10-year period. ${ }^{[12]}$ This retrospective survey, the largest reported, confirms an overall high success rate, with no patient dying of a hypoxic cardiac arrest during airway management. Of note, a mere 21 patients required an emergency cricothyroidotomy or tracheotomy ${ }^{[12]}$ Despite obvious limitations to this study (retrospective, specialist trauma centre, resource-rich setting), the results are compelling.

In keeping with other international airway guidelines we have purposefully not included a description of anaesthetic induction agents, or indications or preparations for an RSI. ${ }^{[8,9,12]}$ Such additional information would crowd the algorithm unnecessarily and would be counterproductive in achieving clarity. A discussion of the various RSI drugs is best dealt with in a separate guideline.
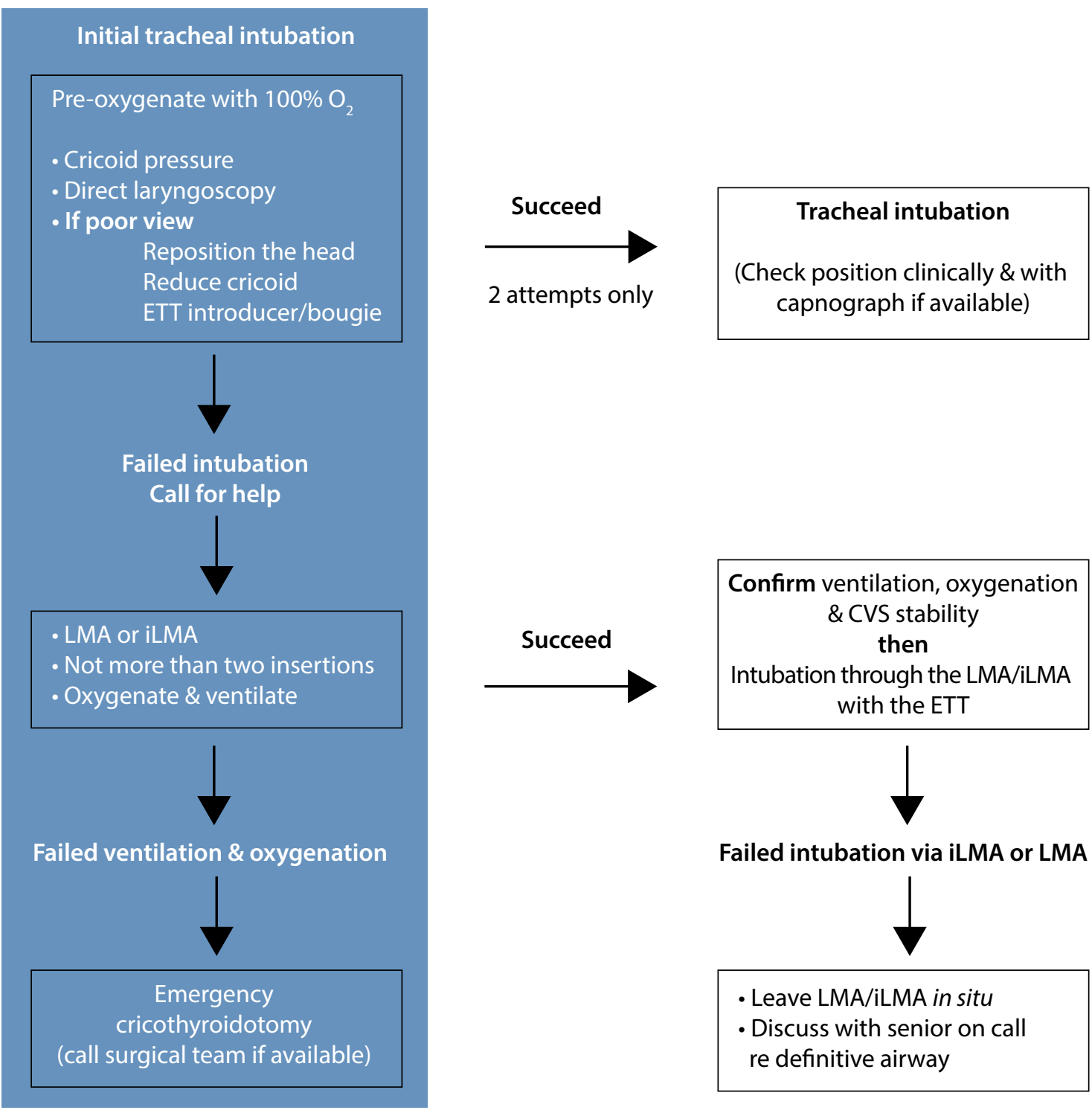
Confirm ventilation, oxygenation \& CVS stability then
Intubation through the LMA/iLMA with the ETT

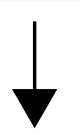

Failed intubation via iLMA or LMA

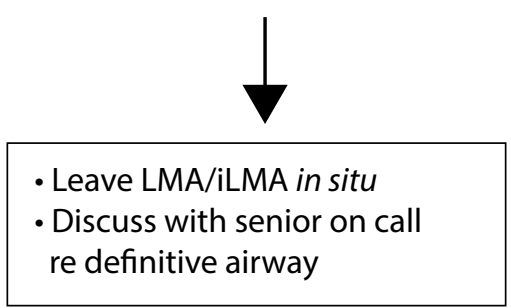

Fig. 1. Trauma airway protocol. $(E T T=$ endotracheal tube; $C V S=$ cardiovascular system; $L M A=$ laryngeal mask airway; iLMA = intubating laryngeal mask airway.) 
Furthermore, our protocol does not aim to be all-encompassing, but maps out a series of critical steps to ensure safe airway management under pressure. Head positioning, for example, remains crucial in the subset of patients in whom it can be undertaken safely (e.g. single gunshot abdomen), but neck extension cannot be recommended when an injury to the cervical spine is suspected. A bougie is a simple, widely available intubation adjunct that often proves helpful and can be used safely with little training. Cricoid pressure in the RSI scenario remains controversial, ${ }^{[14]}$ and in some parts of the world (e.g. mainland Europe) the use of cricoid pressure is no longer routinely recommended. ${ }^{[15]}$ When cricoid technique is poor, it can worsen laryngoscopy views and limit success of intubation. ${ }^{[8,11,12]}$ In the UK, and to a lesser extent in SA, cricoid pressure continues to be part of national guidance, which is why we have included it. ${ }^{[7,8]}$

Calling for help remains an integral part of all emergency airway algorithms. However, in the SA context rural hospitals frequently have only one doctor on site after hours, with limited senior support. We have designed our protocol with this in mind. Should the primary intubation attempt fail, rapid use of an SGA device can be life saving. Although not a definitive airway, an SGA is easy to insert and can improve the patient's gas exchange. ${ }^{[12]}$

SGAs can also be used as a conduit for intubation if the operator feels confident enough, particularly with the intubating laryngeal mask. ${ }^{[8,11,12]}$ It is therefore our view that given the precarity of the skills on the ground and the clinical condition of these patients, an SGA is life saving. ${ }^{\left[{ }^{[12]}\right.}$ The aim should be oxygenation and ventilation with the final endpoint of a safely inserted secure airway.

While emphasising the need for technically simple interventions, the proposed algorithm includes a surgical airway as a last resort. Unfortunately, it is a skill that most doctors will have very little opportunity to practise. Nonetheless, it remains a vitally important intervention that all doctors dealing with emergency airways need to be prepared to carry out. If SGA placement is not successful, an emergency cricothyroidotomy or tracheotomy is performed immediately in order to secure the patient's airway.

\section{Conclusion}

Management of the emergency trauma airway is difficult. As pointed out, haemodynamic compromise, cervical immobility, direct airway trauma, oropharyngeal bleeding and the need for immediate action all complicate airway management. ${ }^{[6,11]}$ Trauma patients who need an emergency airway intervention require rapid and decisive decisionmaking in the face of incomplete information and management via a preplanned airway algorithm that includes rescue techniques in the event of failure. ${ }^{[6]}$

Despite many useful publications in the literature, there seems to be an absence of concise, practical point-of-care guidelines to the lowest common denominator, i.e. the junior doctor. ${ }^{[6-9]}$ We hope that our emergency airway protocol addresses some of these shortcomings and assists junior doctors to provide the best care possible for trauma patients under difficult circumstances. We urge SA medical bodies to consider this algorithm, designed for rural healthcare practitioners with minimal experience, when drawing up future guidelines on airway management.

1. Norman R, Matzopoulos R, Groenewald P, Bradshaw D. The high burden of injuries in South Africa. Bull World Health Organ 2007;85(9):695-702. [http://dx.doi.org/10.2471/BLT.06.037184]

2. Hardcastle TC, Samuels C, Muckart DJ. An assessment of the hospital disease burden and the facilities for the in-hospital care of trauma in KwaZulu-Natal, South Africa. World J Surg 2013;37(7):1550-1561. [http://dx.doi.org/10.1007/s00268-012-1889-1]

3. Healthcare for London: Incidence of major trauma. NHS London, 2011. http://www.londonhp.nhs.uk/ wp-content/uploads/2011/03/Major-Trauma-Incidence.pdf (accessed 16 November 2013).

4. Hardcastle TC, Finlayson M, van Heerden M, Johnson B, Samuel C, Muckart DJ. The prehospital burden of disease due to trauma in KwaZulu-Natal: The need for Afrocentric trauma systems. World J Surg 2013;37(7):1513-1525. [http://dx.doi.org/10.1007/s00268-012-1852-1]

5. Hardcastle TC, Goff T. Trauma unit emergency doctor airway management. S Afr Med J 2007;97(9):864-867.

6. Horton CL, Brown CA 3rd, Raja AS. Trauma airway management. J Emerg Med 2014:46(6):814-820. [http://dx.doi.org/10.1016/j.jemermed.2013.11.085]

7. Emergency Medicine Society of South Africa Practice Guideline EM015. Rapid sequence intubation. . Emergency Medicine Society of South Africa Practice Guideline EM015. Rapid sequence
EMSSA, 2010. http://emssa.org.za/documents/em017.pdf (accessed 24 November 2013).

8. UK Difficult Airway Society Guidelines. Rapid sequence induction guidelines. UK Difficult Airway ociety, 2006. http://www.das.uk.com/guidelines/rsi.html (accessed 24 November 2013).

9. Apfelbaum JL, Hagberg CA, Caplan RA, et al. Practice guidelines for management of the difficult airway: An updated report by the American Society of Anesthesiologists task force on management of the difficult airway. Anesthesiology 2013;118(2):251-270. [http://dx.doi.org/10.1097/ ALN.0b013e31827773b2

10. Petrosoniak A, Hicks CM. Beyond crisis resource management: New frontiers in human factors training for acute care medicine. Curr Opin Anaesthesiol 2013;26(6):699-706. [http://dx.doi. org/10.1097/ACO.0000000000000007]

11. Langeron $\mathrm{O}$, Birenbaum A, Amour J. Airway management in trauma. Minerva Anesthesiol 2009;75(5):307-311

12. Stephens CT, Kahntroff S, Dutton RP. The success of emergency endotracheal intubation in trauma patients: A 10-year experience at a major adult trauma referral center. Anesth Analg 2009;109(3):866872. [http://dx.doi.org/10.1213/ane.0b013e3181ad87b0]

13. Emergency Medicine Society of South Africa Practice Guideline EM004: Emergency Centre Emergency Medicine Society of South Africa Practice Guideline EM004: Emergency Centse
Equipment. EMSSA, 2008. http://emssa.org.za/documents/em004.pdf (accessed 12 December 2013).

14. Butler J, Sen A. Best evidence topic report. Cricoid pressure in emergency rapid sequence induction. Butler J, Sen A. Best evidence topic report. Cricoid pressure in emergency rapid
Emerg Med J 2005;22(11):815-816. [http://dx.doi.org/10.1136/emj.2005.030205]

15. Theiler L, Fischer H, Voelke N, Basciani R, Hasty F, Greif R. Survey on controversies in airway management among anaesthesiologists in the UK, Austria and Switzerland. Minerva Anestesiol 2012;78(10):1088-1094.

Accepted 14 May 2014. 